\title{
Performance Analysis for Visual Data Mining Classification Techniques of Decision Tree, Ensemble and SOM
}

\author{
C. M. Velu \\ Research Scholar, Faculty of Information and \\ Communication Engineering, Anna University, \\ Chennai - 600129, Tamil Nadu, INDIA
}

\author{
Kishana R. Kashwan, IEEE Member \\ Department of Electronics and Communication \\ Engineering-PG, Sona College of Technology, TPT \\ Road, Salem - 636005, Tamil Nadu, INDIA
}

\begin{abstract}
This research paper is a comprehensive report on experimental setup, data collection methods, implementation and result analyses of market segmentation and forecasting using neural network based artificial intelligence tools. The main focus of the paper is on visual data mining applications for enhancing business decisions. The software based system is implemented as a fully automated and intelligent enough to take into effect of each sales transaction. It updates and instantly modifies forecasting statistics by receiving input sales data directly from sales counter through networked connectivity. The connectivity may be wired or wireless. Three artificial intelligence tools, namely decision tree, ensemble classifier and Self Organizing Maps (SOM) are used for data processing and data analysis. The visual data mining concept is implemented by presenting results in the form of visual interpretation in as simple as possible way to understand very complex statistics. The current research results are mapped to interactive visualization by using multilevel pie charts, multi bar charts, histograms, scatter plots, tree maps and dataflow diagrams. The different visualization techniques help in understanding different levels of information hidden in very large data sets. The results analysis show that decision tree has classified data correctly up to a $86.0 \%$, ensemble techniques produced an average of $88.0 \%$ and the predictions using SOM has accuracy of $90.0 \%$. The survey carried out after implementation and use of the system shows that the system is very easy to understand and can be interpreted quickly with minimum efforts.
\end{abstract}

\section{General Terms}

Computer science and engineering, information technology, data mining, market, business.

\section{Keywords}

Visualization techniques, visual data mining, neural networks, decision tree, forecasting, business decision.

\section{INTRODUCTION}

The classification is very important step in intelligent systems such as market forecasting, language processing, data mining, interpretation of useful information etc. [1]. Classification is also an integral part of machine learning such as support vector machine and self organizing maps [2]. Due to sudden and exponential development of information technology in recent times has created easy and quick ways to collect huge data of any quantum. This has definitely made much more comfort for researchers to collect data. There has been tendency to have huge data collection for any experimental analysis to conclude more confidently. On the one hand the information technology has contributed much for automation on data collection and on the other hand it has posed a big challenge that how to interpret such a huge data meaningfully [3]. There can be very small but useful information or knowledge hidden in such a vast pool of data. Extractions of useful knowledge from huge data sets need different techniques and strategies. These techniques are desired to be faster, more accurate and above all very intelligent. Representation of useful information extracted from massive data may be done in many ways but one such technique called visualization has become very popular due to its instantaneous interpretational characteristics [4].

The visualization bridges the user to important information by mental interfacing. The user can understand quite complex statistics very easily by just looking at some of the visuals such as graphs, figures, maps and tables. It is very critical to present visuals in such a way that it interprets information in a most comprehensive manner. This is not quite simple and need many computational steps before a meaningful visual is created. Any good visual will aid human understanding power and thus create an opportunity to use the knowledge for optimization of business solutions such as market segmentation and forecasting [5]. The visualization also helps in acquiring knowledge more comprehensively and most important, very quickly [6]. It conveys information in very concise and portable format for different levels of details.

Visualization involves mapping of the data into some types of drawing or graphical objects. The accuracy in mapping is most important and critical to maintain originality of the information created by intelligent models. The relevant and precisely required data must be chosen for visual objects so that redundant data is eliminated. Choosing methods of visual objects to fit the available space and time constraints is equally very important. Interaction oriented visual objects provide additional flexibility to user to modify visualization easily so that user may explore 'if then...' possibility and related outcomes of that effect. This flexibility is itself a prediction under constraints and conditions, which is very important for business exploration. Visualization is quite often very helpful in designing models for practical solutions of complex problems [7].

There are many categories of visualization methods [3]. There are two visualization methods which are within the scope of this research. These are data visualization and information visualization. The former involves in presenting data by schematic or diagrammatic forms such as charts, histograms, tables and scatter plots. The latter involves presentation of information by graphical objects so that it interprets information, provides matching solutions, helps in 
identification of patterns available in data, indicates changes and the direction of changes in massive data pools and finally enhances cognitive perception.

This research is aimed at finding business solutions for forecasting and market segmentations by using neural networks and then representing data processing along with result analyses in visualization form. Furthermore, it emphasizes to present information to user for several levels of details such as simple knowledge to strategic and analytical descriptions [7].

Data mining is normally used for market segmentations and forecasting of sales projections. Data mining is software based intelligent technique used to collect customer information for Customer Relationship Management (CRM) in many business organizations. [8]. Management skills can be enhanced by data mining techniques for statistical prediction models greatly. Data mining theory has been used for customer segmentation in retail industries to improve business strategies [9]. It is very important for business organizations to continuously add on new customers. Information technology and data mining based methods are useful for forecasting [10]. Data mining is used for knowledge classification and searching relevant and useful information from collected data. This improves information management process.

Data mining techniques can determine customer information by analyzing sales data [11]. This can be done quickly. The business predictions require quite often the future for long term planning and forecasting [12]. Many individual prediction models have shown little success whereas data mining techniques are more accurate in forecasting customer lifetime value [12]. The recent research works indicate that data mining along with 3-dimensional data visualization is very helpful in quick decisions and understanding of complex business issues easily. The 3-dimensional visualization is used to slice, rotate and zoom in the data projections to obtain minute details by visual models and objects. Business management can be represented by customer knowledge management [13]. The dynamic customer behavior can be modeled with the help of customer sales data [14].

CRM improves business environment by predicting customer's behavior [15]. The successful business enterprises have past records of customer preferences by keeping their up to date knowledge [16]. For promoting business, it requires modern information technology based tools. The integrated models of data mining and visualization techniques are more powerful and intelligent enough to predict better. Adaptive models are more suitable for complex and dynamic business. Visualization of information can be used as joint strategy of sales and marketing which requires understanding the customers' behavior in contrasting situations [17].

\section{IMPLEMENTATION OF ARTIFICIAL INTELLIGENT MODELS}

These are basically neural network based techniques which are implemented by software programming and hardware circuits by mimicking human brain in general and neurons in particular for learning process. Neural network models work much similar to neurons and neuro-transmitters in human brain. The decision making process of artificial neural networks is based on past knowledge and current conditions of rule based functions. There are many types of neural networks and techniques used for different intelligent applications. This research has been focused on three main tools of artificial intelligence. These tools are briefly described in the following sub headings.

\subsection{Decision Tree Model}

Decision tree is based on a tree-like graphical and hierarchical structure for taking decisions along subsequent series paths. There can be number of parameters which may be considered for decision making such as cost or length. In multi branching tree, a decision may be taken based on the cost value of node's branches [16]. Decision tree is suitable for selecting independent variables. It identifies categories or intervals which are widely different. It allows selecting additional variables by inserting additional branch if there is large difference among variables already chosen. One of the most important feature of decision tree is to find out some of the relationships in data sets, which otherwise may not be explicit in nature. The other use of decision tree is, sometimes, to correctly fit missing data.

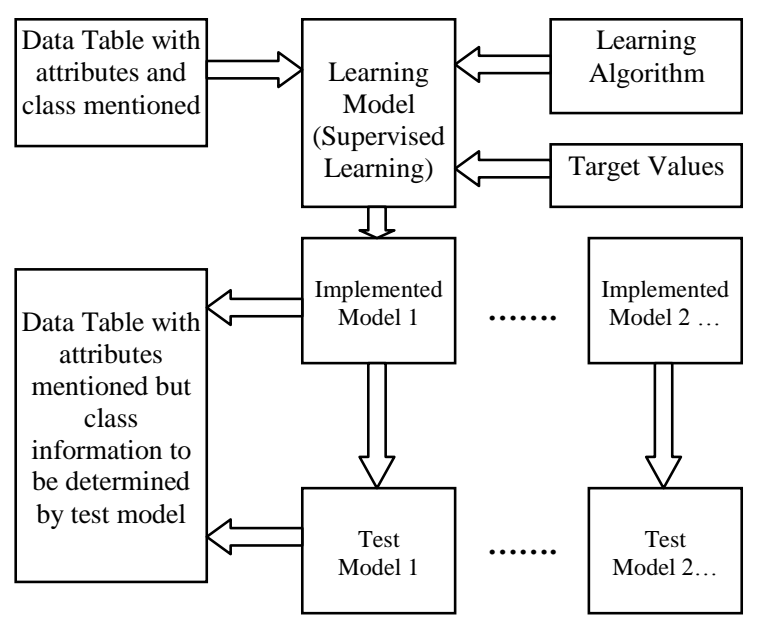

Figure 1: General classification model for predicting class of the data set

The basic classification task is illustrated by Figure 1 . The training data is used to train the model using supervised or unsupervised learning styles according to the algorithms chosen for training. The algorithm describes sequence and step by step procedure to accomplish training of the model. Target values remind model whether it is correctly producing outputs, thus target value acts as a supervisor. A learnt model is now ready to be tested on another set of data with some of the features to be determined. In current example, as shown in Figure 1, the training data has class a mentioned but test data has to be assigned a class by trained model by perdition. Thus the classification model, after training, predicts class of the data during test phase.

Based on the classification model as described in Figure 1 above, a decision tree is constructed. The decision tree explains how decision is taken for classifying or indicating the category of the problem under investigation. Figure 2 illustrated decision tree process. Training data has features which are determined by using data preprocessing techniques and feature selection criteria. A class called potential customer is determined by decision model based on the attributes present in data sets. The attributes are indicated in Table 1 as shown below. 
Table 1. Training data for decision tree test model

\begin{tabular}{|l|c|c|c|c|c|}
\hline S1. No. & $\begin{array}{c}\text { Value of } \\
\text { electrical bill } \\
\text { in INR } \\
\text { (Continuous) }\end{array}$ & Life Insurance & House Loan & $\begin{array}{c}\text { Income in } \\
\text { INR } \\
\text { thousands } \\
\text { (Continuous) }\end{array}$ & $\begin{array}{c}\text { Class } \\
\text { (Potential } \\
\text { Customer) }\end{array}$ \\
\hline 1. & 1750 & YES & NO & $150 \mathrm{k}$ & NO \\
\hline 2. & 1580 & YES & YES & $160 \mathrm{k}$ & NO \\
\hline 3. & 1180 & NO & NO & $165 \mathrm{k}$ & YES \\
\hline 4. & 1500 & YES & YES & $145 \mathrm{k}$ & NO \\
\hline 5. & 1350 & NO & NO & $195 \mathrm{k}$ & YES \\
\hline 6. & 1480 & NO & YES & $178 \mathrm{k}$ & YES \\
\hline 7. & 1900 & YES & YES & $197 \mathrm{k}$ & YES \\
\hline 8. & 1340 & NO & NO & $145 \mathrm{k}$ & YES \\
\hline 9. & 1600 & NO & YES & $136 \mathrm{k}$ & NO \\
\hline 10. & 1920 & YES & YES & $160 \mathrm{k}$ & NO \\
\hline
\end{tabular}

The decision tree is split into attributes such as electricity bill in continuous range, absence or presence of insurance and house loan etc. Based on trained model, the test model has to predict the class. The class in this case is to classify the customer as a potential customer or non-potential customer.
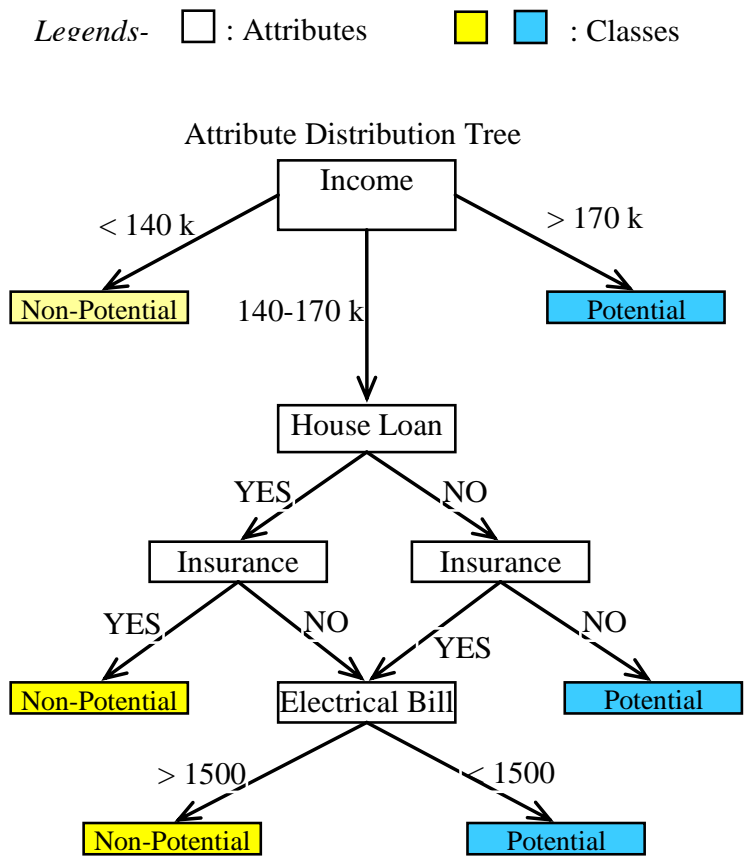

Figure 2: Decision tree flow diagram for classification

The classification is done based on the attributes' values and yet in other cases simply by presence or absence of the attributes. The model developed is representative of few sample attributes selected as shown in Figure 2. The actual research has taken into account large number of attributes and large data samples. Nearly half of the data sets are used for training purpose and remaining half data sets are used for testing of the model. Figure 2 gives very clear visualization perception to understand classification of customers by using attribute paths showing values and absence or presence features. It finally predicts classes of customers as non potential customers or potential customers shown by color codes of orange and blue respectively.

\subsection{Ensemble Model}

These are various learning algorithms that are used for training of neural network models. Such trained neural network models are used for classification of data sets by taking a vote of classification from each of the individual algorithms [18]. Bayesian averaging is such an example of ensemble model. The scope of this research is limited to only bagging algorithm for ensemble model implementation. Sometimes it is not possible to have many data sample collections. Also it is not always wise to assume certain data distributions. Bagging can be useful under such conditions as it produces multiple input data sets by replicating and then making different combinational training sets by bootstrapping. The bootstrapping is performed by randomly selecting a relatively smaller set of data from large aggregates of data and then classification is done. In next step some of the data sets are replaced from aggregate data sets to make a new set of training data set. Some of the data sets may be repeated.

\section{Table 2. Bagging algorithm - execution steps flow}

1. Divide data sets randomly into two sets, each one for training and testing of the model respectively

2. Get input data of training set and generate bootstrap samples by repeating some of the sets and replacement for next step

3. Compute bootstrapped estimated predictions

4. Repeat step 3 for $n$ times (where $n$ is randomly selected any large number)

5. Construct a classification tree and validate by test data set for error rate

6. Estimate the class of input data. If there is a tie, estimate the class with the lowest class threshold.

7. Repeat steps 1 to $6, N$ times by randomly dividing training data sets where $N$ is arbitrary selected number for aggregating $N$ times to reduce prediction errors.

Bagging is a learning algorithm wherein multiple predictions are generated and subsequently aggregated by averaging. The multiple predictions are produced by bootstrap replicates of learning data sets. The replicates are, then used for new learning sets [19]. If a small noisy disturbance in neural learning causes large deviations in predictions, then bagging can improve prediction accuracy significantly. The term bagging is derived as an acronym of bootstrap aggregating. The execution steps of bagging algorithm can be summarized as given in Table 2. Ensemble model is normally more accurate than any of the individual classifier of the same ensemble. One of the big challenges in supervised learning of neural networks is local optimal solution. Once an algorithm is struck at local optimal value, it repeats and may not converge forever in trying to explore global optimal solution. Ensemble may be used to start learning from many local points to provide better approximation of the solution. Table 3 shows that how some of the original data sets are replicated and replaced to form subsequent 3 sets of new data for 
training neural networks. Alternatively, new data sets can also be formed by keeping smaller number of data points in each data set and randomly selecting data from original data.

Table 3. Formation of different data sets for bagging algorithm based supervised learning

\begin{tabular}{|c|c|c|c|c|c|c|c|c|c|}
\hline $\begin{array}{c}\text { Original } \\
\text { data }\end{array}$ & 15 & 25 & 35 & 45 & 55 & 65 & 75 & 85 & 95 \\
\hline Set-1 & 25 & 35 & 25 & 55 & 65 & 35 & 45 & 25 & 85 \\
\hline Set-2 & 15 & 25 & 15 & 95 & 35 & 35 & 45 & 35 & 15 \\
\hline Set-3 & 35 & 25 & 45 & 25 & 35 & 95 & 75 & 35 & 25 \\
\hline
\end{tabular}

In an ensemble individual decision from each of the classifiers is combined with or without weighted voting for classifying new data sets. Supervised learning quite often uses ensembles for classification. This experimental work is focused on market segmentation and forecasting solutions for sales data. A number of attributes and variables are chosen and then bagging principles are applied to predict the sales projections over a period of time. Table 4 shows the sample results of ensemble technique of bagging. The experimental data sets are collected online by automated software based model. Almost $50 \%$ of the data sets are used for training and then remaining data sets are used to test the model.

Table 4. Classification results of ensemble model

\begin{tabular}{|c|c|c|c|c|c|}
\hline $\begin{array}{c}\text { Data } \\
\text { Set. }\end{array}$ & $\begin{array}{c}\text { Number of } \\
\text { Samples }\end{array}$ & Training Data & Test Data & $\begin{array}{c}\text { Number of } \\
\text { Variables }\end{array}$ & $\begin{array}{c}\text { Classes } \\
\text { Observed }\end{array}$ \\
\hline 1. & 325 & 160 & 165 & 15 & 3 \\
\hline 2. & 321 & 160 & 161 & 14 & 4 \\
\hline 3. & 243 & 120 & 123 & 17 & 2 \\
\hline 4. & 372 & 180 & 192 & 20 & 3 \\
\hline 5. & 312 & 160 & 152 & 16 & 4 \\
\hline 6. & 341 & 170 & 171 & 17 & 3 \\
\hline
\end{tabular}

As shown in Table 4, ensemble technique identified classes in the data sets correctly. Replication and replacements are made for generating new test training data.

\subsection{Self Organizing Map Model}

These maps are neural networks based on the unsupervised learning process. One such example is competitive learning in which output neurons compete with each other for output. Only one neuron gets activated at a time and is called winner neuron. Normally lateral connections are provided for negative feedback which acts as inhibition connection. In this process of competitive and unsupervised learning, neurons get organized by themselves. Thus, the name self organizing maps (SOM) have come in. These maps are based on the principles of neuroscience that spatial location of an output neuron corresponds to a feature present at input space. The main working principle of SOM is to transform input patterns by mapping at output at fewer discrete dimensions than the input dimensions [20]. In fact SOM reduces dimensional size greatly. The neurons become organized to various input patterns by way of competitive learning. SOM makes topographic maps for input patterns. These maps are nonlinear in nature.

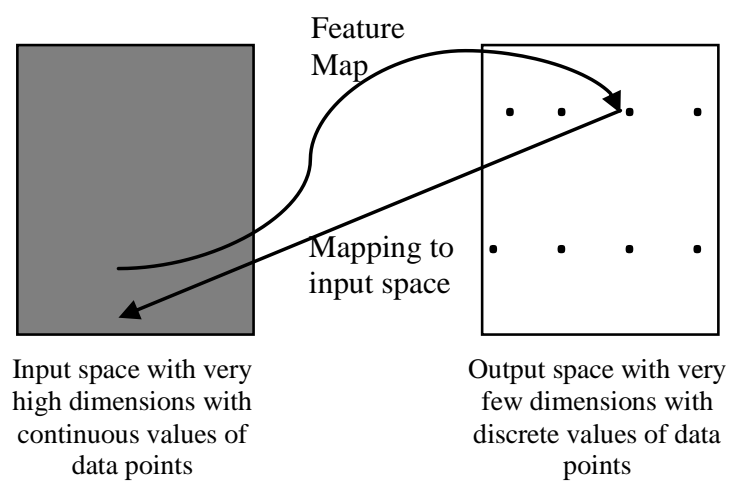

Figure 3: Visualization topology of SOM - a point in input space is mapped to output space in accordance to feature of selections. Similarly each point in output space is mapped to input space

For this research, a particular type of SOM called Kohonen Network is used for forecasting and market segmentation problem. These networks have feed forward structure and an output layer of neurons called computational layer. Each neuron in output computational layer is fully connected to input layer.

Table 5. SOM algorithm - execution steps flow

1. Initialize connection weights with random values at beginning and set suitable learning rate.

2. For each input pattern, compute discriminant function value for each neuron.

3. Declare a neuron as winner which has smallest discriminant function value.

4. Winner neuron determines location of excited neighborhood neurons.

5. Excited neurons adjust their values by reducing discriminant function value through connection weights for making stronger of winner neuron for similar future computations.

The discriminant function is square of Euclidian distance between the input vector and the weight vector for each neuron. Thus the neuron whose weight vector is closely matched with input vector is declared winner. Similarly, all other neurons of output layer are mapped to the neurons of input layer. The winner neuron is also called firing neuron. If a neuron fires, the neighboring neurons get excited laterally but the excitation is limited to within small neighborhood. In each epoch learning weight matrix is updated in such a way that winning neuron and its neighboring neurons' weight vectors move towards input vector.

The training of SOM can be explained as initially output neurons are mapped to some randomly selected data points of input space in its first epoch. During next epoch, weight matrix is updated in such a way that weight vector of winning 
neuron moves towards a data point with minimum Euclidean distance. This process continues until convergence occurs. The training concludes at convergent point. SOM are prone to metastable state where it may not converge. Initial parametric value selection may be critical for metastable state, but SOM can start at any point just selected randomly. General visualization of SOM architecture is illustrated in Figure 4. It shows input-output mapping for computational layer. Each of the output is mapped back at the source input. The central neuron with maroon color is depicted as winner neuron. Immediate neighboring neurons of winner neuron are excited slightly only in output space as indicated by color codes.

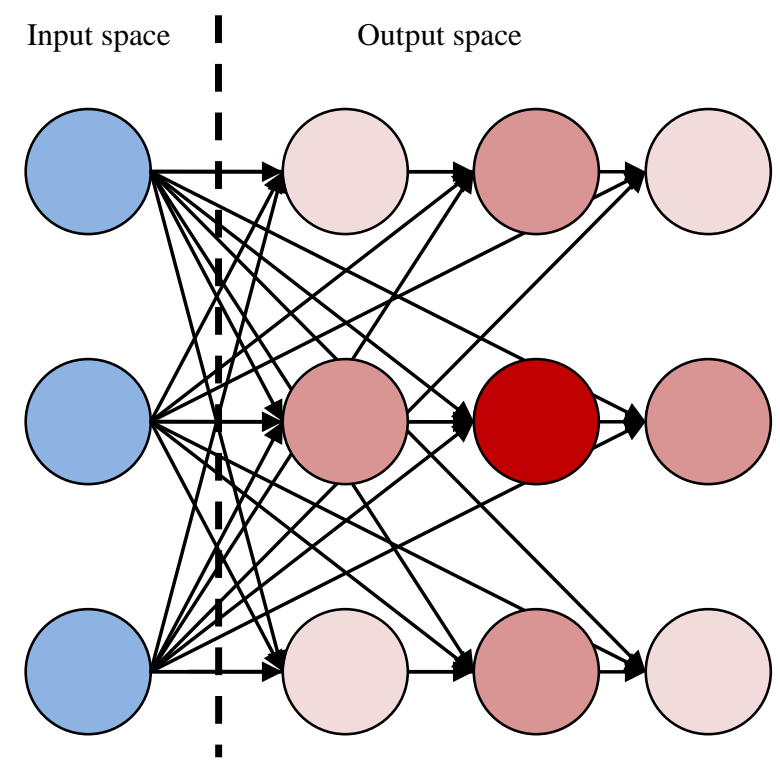

Figure 4: Visualization of input-output mapping architecture of SOM

The experimentation of the applying SOM for the classification of the customers into different classes is shown in Table 6. Data collection is done directly from sales counters for SOM analysis. A total of 6 data sets, each consisting of 240 to 370 data points are compiled. Nearly half of the data points are used to train SOM nets and remaining half of the data points are used to test the model developed to validate the effectiveness and accuracy of the model. The numbers of variables are chosen according to the data sets and desired analysis to be carried out. Variables such as age, education, life style preferences, income range, recreational interests, use of modern electronic gadgets etc. are chosen in such a way that these variables likely to make an effect on customers' behavior for marketing and forecasting. Some of the variables may not be useful in some of the analyses, thus it can be seen in Table 6 that number of variables are different for each data set. For example, $3^{\text {rd }}$ data set, which has 240 data points collected and 13 variables chosen, SOM has found 3 classes namely potential, average and non potential customers. Similarly for $4^{\text {th }}$ data set with 370 data points collected and 14 variables chosen, SOM has observed 5 classes, namely highly potential, potential, average, non potential and insignificant customers. This classification gives quite good insight about customer's behavior for sales projections and forecasting.

The geographical location was also very important for planning new supply stores. The SOM analysis also predicted location wise statistics to find out scattering density of the customers in each of the classes mentioned above. This can help in planning of expansion of business in certain locality.

Table 6. Classification results of SOM model

\begin{tabular}{|c|c|c|c|c|c|}
\hline $\begin{array}{c}\text { Data } \\
\text { Set. }\end{array}$ & $\begin{array}{c}\text { Number of } \\
\text { Samples }\end{array}$ & Training Data & Test Data & $\begin{array}{c}\text { Number of } \\
\text { Variables }\end{array}$ & $\begin{array}{c}\text { Classes } \\
\text { Observed }\end{array}$ \\
\hline 1. & 300 & 160 & 140 & 14 & 3 \\
\hline 2. & 320 & 160 & 160 & 16 & 2 \\
\hline 3. & 240 & 120 & 120 & 13 & 3 \\
\hline 4. & 370 & 180 & 190 & 14 & 5 \\
\hline 5. & 310 & 160 & 150 & 15 & 3 \\
\hline 6. & 340 & 170 & 170 & 19 & 4 \\
\hline
\end{tabular}

Table 7. Overall results of decision tree model, ensemble model and SOM model classification

\begin{tabular}{|c|c|c|c|c|c|c|c|}
\hline $\begin{array}{c}\text { S } \\
\text { No. }\end{array}$ & Data Set & \begin{tabular}{|c|}
$\begin{array}{c}\text { No. of } \\
\text { Attributes }\end{array}$ \\
\end{tabular} & $\begin{array}{c}\text { No. of } \\
\text { data } \\
\text { points }\end{array}$ & $\begin{array}{l}\text { Decision } \\
\text { Tree }(\%)\end{array}$ & $\begin{array}{c}\text { Bagging } \\
(\%)\end{array}$ & $\begin{array}{c}\text { SOM } \\
(\%)\end{array}$ & $\begin{array}{c}\text { Actual } \\
(\%)\end{array}$ \\
\hline 1 & Grocery & 11 & 200 & 87.8 & 91.1 & 90.8 & 93.4 \\
\hline 2 & Cosmetics & 10 & 500 & 83.9 & 89.3 & 89.3 & 85.6 \\
\hline 3 & Edibles & 12 & 1000 & 78.2 & 85.5 & 87.1 & 85.8 \\
\hline 4 & Sanitary items & 14 & 1000 & 87.8 & 90.2 & 90.8 & 92.0 \\
\hline 5 & $\begin{array}{l}\text { Electronic } \\
\text { items }\end{array}$ & 10 & 700 & 76.7 & 80.3 & 80.1 & 79.5 \\
\hline 6 & Toys & 12 & 600 & 89.6 & 80.1 & 83.0 & 87.4 \\
\hline 7 & $\begin{array}{l}\text { Leisure } \\
\text { readings }\end{array}$ & 16 & 200 & 91.2 & 96.0 & 97.4 & 86.3 \\
\hline 8 & Toiletry items & 10 & 500 & 96.7 & 98.6 & 97.3 & 99.0 \\
\hline 9 & Cleaning agent & 12 & 200 & 80.9 & 88.2 & 89.2 & 84.3 \\
\hline 10 & Utensils & 14 & 100 & 75.3 & 77.6 & 83.0 & 78.8 \\
\hline 11 & Fast food & 12 & 300 & 76.3 & 80.6 & 82.6 & 86.4 \\
\hline 12 & Beverages & 32 & 1200 & 85.7 & 97.8 & 97.8 & 95.3 \\
\hline 13 & Readymade & 14 & 700 & 88.8 & 78.1 & 85.9 & 85.2 \\
\hline 14 & Foot wear & 10 & 900 & 96.3 & 97.0 & 98.7 & 99.5 \\
\hline 15 & Healthcare & 12 & 700 & 95.0 & 97.0 & 98.0 & 97.9 \\
\hline \multicolumn{4}{|c|}{ Average values } & 86.0 & 88.0 & 90.0 & 89.0 \\
\hline
\end{tabular}

\section{RESULTS AND DISCUSSIONS}

The overall result analysis for market data are summarized in Table 7. The experimental data sets consists of 15 broad categories of market items for which it is planned to do customer classification and segmentation for sales predictions. Number of data points and number of attributes are chosen depending upon size of business volumes and applicable variables for the broader category-wise groups. The attributes are chosen such as age, gender, educational level, life style preferences, critical requirements, alternative choice and so on. The attributes vary in number from one category of items to another due to characteristics matching with chosen attributes. For example critical requirement may not 
applicable for luxury items and life style habits. Similarly data sets also vary from one category to another. This can be explained as some of the items move fast and has abundance records available in a given time period but same time some other items may not have same business volume. Due to limitations of data availability, variable data sets are collected and analyzed for proposed neural network models.

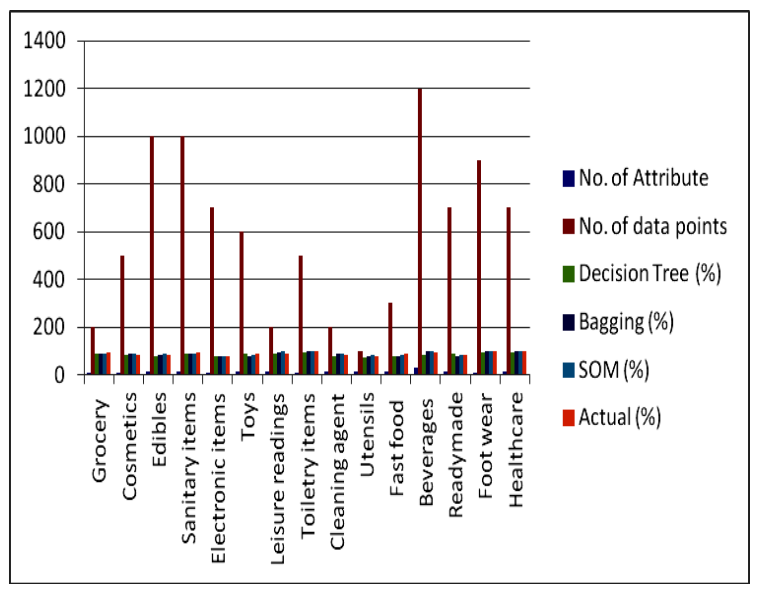

Figure 5: Visualization topology of decision tree model, ensemble model and SOM model for overall performance comparison analyses for market classification predictions

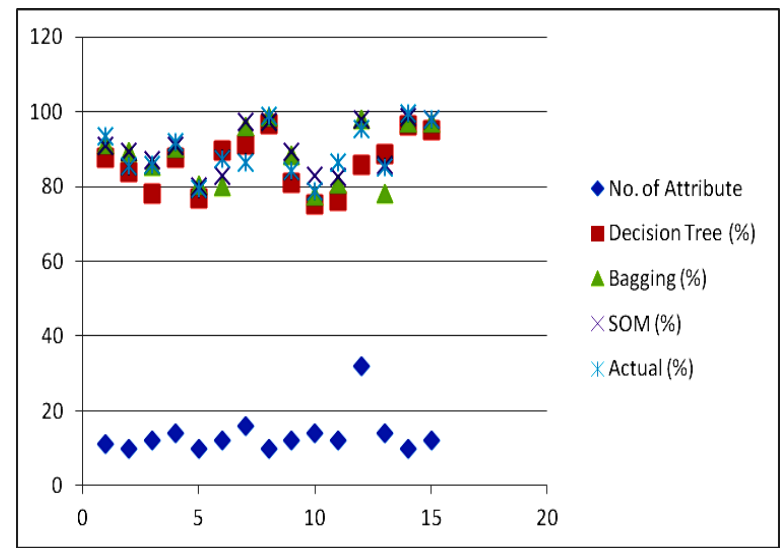

Figure 6: Visualization of \% comparison performance of decision tree model, ensemble model and SOM model

Table 7 shows percentage comparison of correctly classified results of 3 models proposed in this research work. Decision tree model, ensemble model and SOM model has classified customers into appropriate class on an average of $86.0 \%$, $88.0 \%$ and $90.0 \%$ respectively. The results are then compared with actual classes based on customer feedback and manual classification. Manual classification is performed by collecting data using hard records and surveys. Subsequently, various statistical methods employed to compute various statistical quantities. This method of validation is complex and time consuming but generally proven and reliable as it has been used for many decades especially in past in the absence of computing facilities and information technology. The system, however, learns from its own records of sales and updates its knowledge. Thus the proposed system is self evolving and has capability to learn from experience. Actual classification is on an average is $89.0 \%$ which is quite close to the classification rate of neural network models proposed and implemented.

The main focus of this research is emphasized on visualization for quick and easy understanding. The results of Table 7 are illustrated by visuals in Figure 5 and Figure 6 . These visual aids give quick view that which of the three models has classified at what level of accuracy. For given classification current case, bar charts and scatter plots suit more aptly. Accordingly Figure 5 and Figure 6 use the concepts of bar charts and scatter plots respectively. After research results employed for actual practice at sales counters, a survey is carried out to find the ease and effectiveness of the models implemented. Survey reports says $100 \%$ ease of use and $98 \%$ feels that it helps in more than one way at many decision making points. The users feel that continuous use of proposed models will probably make system more acquaint and users become more dependent on the aid provided by models as practice continues. In any way the paradigm shift will take place and such models will become necessity for carrying out day to day business.

\section{CONCLUSION AND FUTURE WORK}

This paper has focused on proposing intelligent models for market classification of customers into suitable classes in order to enhance business decision making process. The models are validated with rigorous experimental training and then subsequently tested for effectiveness. Three models of decision tree, ensemble model and SOM model are applied and tested for customer classification. Results are quite encouraging. Classifications by models quite closely match with that of statistical results. The models are put in practice in real time business analysis and again validated for accuracy and effectiveness.

The future work will involve more intelligent tools such as fuzzy logic and genetic algorithms for making models further more accurate and reliable. More application areas of health monitoring and field management may be included for testing and validating models. The scope of the research appears quite wide and multi-application oriented.

\section{ACKNOWLEDGMENTS}

Authors express their personal gratitude and sincere thankfulness to the experts and domain specialists who contributed for this research and compilation of report in a direct or indirect way.

\section{REFERENCES}

[1] Jiawei Han and Micheline Kamber, 2008, Data Mining Concepts and Techniques, Elsevier, Second Edition.

[2] Luka Furst, Sanja Fidler, Ales Leonardis, 2008, Selection Features for Object Detection Using an AdaBoostCompatible Evaluation Function, Pattern Recognition Letters, Vol. 29, pp. 1603-1612.

[3] Muzammil Khan and Sarwar Shah Khan, 2011, Data and Information Visualization Methods and Interactive Mechanisms: A Survey, International Journal of Computer Applications, 34 (1), pp. 1-13. 
[4] North C., 2005, Towards Measuring Visualization Insight, IEEE Computer Graphics and Applications, 11(4), pp. 443-456

[5] Alfredo R. Teyseyre and Marcelo R. Campo, 2009, An Overview of 3D Software Visualization, IEEE Transactions on Visualization and Computer Graphics, 15 (1), pp. 87-105.

[6] C. Johnson, 2004, Top Scientific Visualization Research Problems, IEEE Computer Graphics and Applications, 24(4), pp. 13-17.

[7] J. J. Thomas and K. A. Cook, 2006, A Visual Analytics Agenda, IEEE Transactions on Computer Graphics and Applications, 26 (1), pp. 12-19.

[8] Huirong Zhang Yun Chen, 2009, An Analysis of the Applications of Data Mining in Airline Company CRM, Fuzzy Systems and Knowledge Discovery, Sixth IEEE International Conference on, Vol 7, pp. 290 - 293.

[9] Huaping Gong Qiong Xia, 2009, Study on Application of Customer Segmentation Based on Data Mining Technology, Future Computer and Communication, FCC, IEEE International Conference on, pp. 167 - 170

[10] Wu Dong Sheng, 2011, Application Study on Banks's CRM Based on Data Mining Technology, Electrical Information and Control Engineering, ICEICE, IEEE International Conference on, pp. $5727-5731$.

[11] Young Sung Cho Keun Ho Ryu, 2008, Implementation of Personalized Recommendation System Using Demographic Data and RFM Method in e-commerce, Management of Innovation and Technology, ICMIT, $4{ }^{\mathrm{TH}}$ IEEE International Conference on, pp. $475-479$.

[12] Lim Chia Yean and Khoo, V.K.T., 2010, Customer relationship management: Computer-assisted Tools for Customer Lifetime Value Prediction, Information Technology, ITSim, IEEE International Symposium, pp. $1180-1185$

[13] Shaw M. J., Subramaniam C., Tan G. W. and Welge M. E., 2001, Knowledge management and Data Mining for marketing, Decision Support Systems, pp. 127 - 137.

[14] Song H.S., Kim J.K. and Kim S.H., 2001, Mining the Change of Customer Behavior in an Internet Shopping Mall, Expert Systems with Applications, 21(3), pp. 157 170 .

[15] Jill Dyche, 2002, The CRM Handbook: A Business Guide to CRM, Addison-Wesley Professional, First Edition.
[16] A. Berson, K. Thearling and S. Smith, 2000, Building DM Applications for CRM, McGraw-Hill.

[17] Alex Sheshunoff, 1999, Winning CRM Strategies, ABA Banking Journal, pp. 54 - 66.

[18] Thomas G. Dietterich, 2000, Ensemble Methods in Machine Learning, ACM Proceedings of the First International Workshop on Multiple Classifier Systems, pp. 1-15.

[19] Rui Xia, Chengqing Zong, Shoushan Li, 2011, Ensemble of feature sets and classification algorithms for sentiment classification, Information Sciences, 181, pp. 1138-1152.

[20] B. H. Chandra Sekhar and G. Sobha, 2009, Classification of documents using Kohonen's self-organizing map, International Journal of Computer Theory and Engineering, 1(5), pp. 610-613

\section{AUTHORS' PROFILES}

Professor C. M. VELU, received his M.E in CSE from Sathyabama University. He is currently pursuing his doctoral program under the faculty of Information and Communication Engineering registered at Anna University Chennai, India. He has visited UAE as a Computer faculty. He served as faculty of CSE for more than two and half decades. He has published many research papers in international and national journals. His area of interest is Data Warehousing and Data Mining, Artificial Intelligence, Artificial Neural Networks, Digital Image Processing and Pattern Recognition.

Professor Dr. K. R. Kashwan has received the degrees of M.Tech. in Electronics Design and Technology and Ph.D. in Electronics and Communication Engineering from Tezpur University (a central university of India), Tezpur, India, in 2002 and 2007 respectively. Presently he is a Professor and DEAN (Post Graduate Studies) in the department of Electronics and Communication Engineering (Post Graduate Studies), Sona College of Technology (Autonomous), Salem - 636005, India. He has published extensively at international and national level and has travelled to many countries. His research areas are VLSI Design, Communication Systems, Circuits and Systems and SoC / PSoC. He is also director of the Centre of Excellence in VLSI Design and Embedded SoC at Sona College of Technology. He is member of Academic Council, Research Committee and Board of Studies of Electronics and Communication Engineering at Sona College of Technology. He has successfully guided many scholars for their master's and doctoral theses. Prof. Kashwan has completed many funded research projects. Currently, he is working on a few funded projects from Government of India. 